\title{
A simple analytic theory for the statistics of avalanches in sheared granular materials
}

\author{
Karin A. Dahmen ${ }^{1 \star}$, Yehuda Ben-Zion ${ }^{2}$ and Jonathan T. Uhl ${ }^{3}$
}

\begin{abstract}
Slowly sheared granular materials at high packing fractions deform via slip avalanches with a broad range of sizes. Conventional continuum descriptions ${ }^{1}$ are not expected to apply to such highly inhomogeneous, intermittent deformations. Here, we show that it is possible to analytically compute the dynamics using a simple model that is inherently discrete. This model predicts quantities such as the avalanche size distribution, power spectra and temporal avalanche profiles as functions of the grain number fraction $v$ and the frictional weakening $\varepsilon$. A dynamical phase diagram emerges with quasi-static avalanches at high number fractions, and more regular, fluidlike flow at lower number fractions. The predictions agree with experiments and simulations for different granular materials, motivate future experiments and provide a fresh approach to data analysis. The simplicity of the model reveals quantitative connections to plasticity and earthquake statistics.
\end{abstract}

Slip avalanches in slowly sheared granular materials, such as sand and powders, are important for many industrial, engineering, and geophysical processes. Understanding and predicting the dependence on packing fraction, shear rate, and frictional properties are the questions addressed here.

In contrast to traditional models based on continuum mechanics ${ }^{1}$ or on simulations of each individual grain, we use an analytical, discrete, coarse-grained approach. We analytically derive predictions for the statistical properties of slip avalanches at slow shear rates, where grain inertia is negligible (the 'quasi-static' regime). (We do not consider the regimes where grain inertia is non-negligible, such as granular gases $\left.{ }^{2,3}\right)$. Previous studies focused on jamming ${ }^{4,5}$, force chains ${ }^{3,5-9}$, stress drops during avalanches (refs 10-12; P. Yu, T. Shannon, B. Utter \& P. R. Behringer, unpublished data and R. P. Behringer, private communication), and shear localization in shear bands $s^{1,2,13,14}$. Here, we consider a simple model for slip avalanche statistics.

We model the simplified system on a coarse-grained scale (larger than the grain diameter) with a lattice of sites that can either stick or slip under shear. The lattice is either two- or three-dimensional. It has linear extent $L$ and $N=L^{d}$ sites, where $d$ is the dimension of the lattice. $N_{\text {occ }}$ sites are occupied by grains and $N-N_{\text {occ }}$ sites are empty (voids). The 'grain number fraction', $v \equiv N_{\text {occ }} / N$ is proportional to the rescaled packing fraction $\Phi / \Phi_{\max }$, with $v=1$ for the densest possible packing $\Phi=\Phi_{\max }$. Initially all sites are stuck at random initial stresses. We apply a slow shear strain rate by moving one boundary of the lattice at a very slow parallel velocity $V$ (see Fig. 1). This leads to a slow increase of shear stress at each lattice point. A site $i$ slips in the shear direction when its local shear stress $\tau_{i}$ exceeds a random static 'frictional' failure stress $\tau_{s, i}(i=1, \ldots, N)$. (The shape of the narrow distribution of the $\tau_{s, i}$ does not affect the behaviour on long length scales ${ }^{15}$.) A failing
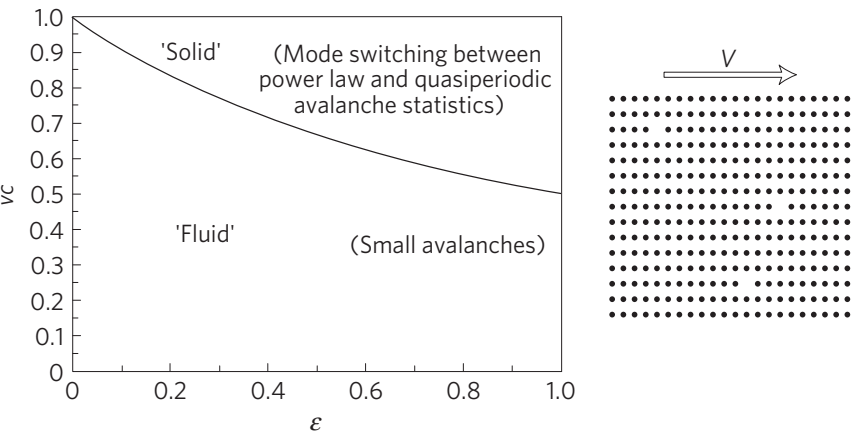

Figure 1 | Analytically computed dynamic 'mean field phase diagram'. Left: Granular materials can respond like a solid or more like a fluid under slow shear, depending on the grain number fraction $v$ multiplied by $c=J /\left(J+K_{L}\right) \approx 1$, and the frictional weakening $\varepsilon$. The bold line is the phase boundary $c v^{*}(\varepsilon)=1 /(1+\varepsilon)$ separating the solid phase from the fluid-like phase. At $c v=1$ and $\varepsilon=0$ is a critical point (see Table 1). Right: Sketch of the sheared model lattice with voids (that is, $v<1, \Phi<\Phi_{\max }$ ). The 'linear system size $L^{\prime}$ is the length of the edges of the (square) granular region in the figure.

site slips during one time step until its stress is relaxed to a local 'arrest stress' $\tau_{a, i}<\tau_{s, i}$. It then resticks, with a weakened, dynamic failure threshold ${ }^{16}$ of $\tau_{d, i} \equiv \tau_{s, i}-\varepsilon\left(\tau_{s, i}-\tau_{a, i}\right)>\tau_{a, i}$. Here $\varepsilon \geq 0$ is a 'weakening parameter' that quantifies the difference between effective static and dynamic 'friction' on meso-scales (larger than the contact level $)^{14,17,18}$. Weakening is associated with dilation, the evolution of frictional resistance, and other effects. It could presumably be tuned by changing the shape and surface of the grains and the packing fraction. A slipping site can trigger other sites to slip in the next time step and so on. The slip avalanche stops when the stresses at all sites are below their current failure thresholds. All failure thresholds then reheal to their static values $\tau_{s, i}$. The material continues to be slowly sheared until a new avalanche starts. (Shear rates faster than the rehealing rate may lead to shear band formation. Here we assume the shear band spans the entire modelled region ${ }^{12}$.)

To solve the model we make the following simplifying assumptions: (1) We study the 'steady state', when all memory of the initial conditions has decayed. (2) It is known that at high packing fractions granular materials have long force chains that often span the entire system ${ }^{3,5-9}$. Long force chains facilitate long range interactions. We approximate them by infinite-range mean field (MF) coupling $J$ between the lattice sites (see Supplementary Information). Mean Field Theory (MFT) effectively averages out the spatial dependencies. (MFT also correctly describes slips in

\footnotetext{
${ }^{1}$ Department of Physics, University of Illinois at Urbana Champaign, 1110 W. Green Street, Urbana, Illinois 61801, USA, ${ }^{2}$ Department of Earth Sciences, University of Southern California, Los Angeles, California 90089-0740, USA, ${ }^{3} 450$ Las Lomas, Dr., La Habra, California 90631, USA. *e-mail:dahmen@illinois.edu.
} 


\section{Table 1 | Comparison of MFT results (power law exponents and scaling behaviour) with numerical simulations and experiments.}

\section{Power law distribution or other universal quantity for densely packed \\ grains (that is near $v c=1$ and $\varepsilon=0$ )}

Mean field theory exponent
Granular simulations ${ }^{14,17,18}$
Avalanche size distribution $D(s) \sim s^{-\kappa}$ Avalanche duration distribution $\sim T^{-\alpha}$

Power spectrum $P(\omega) \sim \omega^{-\phi}$

Source time function $\langle V(t, T)\rangle$ averaged over all avalanches of same duration $T$. Quasi-periodic event statistics (that is, stick slip behaviour)

Mode switching (between power law and quasi-periodic/stick-slip)

\author{
$\kappa=1.5$ \\ $\alpha=2$ \\ $\phi=2$ if $v \approx 1 ; \phi=0$ if $v \ll 1$ \\ Symmetric (parabola)
}

Yes, if $\varepsilon>0$ and $v>v^{*}$

Yes, if $\varepsilon>0$ and $v>v^{*}$ $\kappa=1.5$

$\alpha=2$ or exponential?

$\phi=1.8-2.5,2$

Symmetric (parabola)

Yes, sometimes

Yes, sometimes $\phi=2$ if solid $\phi=0$ if fluid Symmetric: fit by sine function or parabola (?)

Yes, in mode switching regime

Yes, in solid regime

Results that are not yet available from experiments or simulations are indicated by a dash. The agreement with published experiments and simulations is remarkably good. The distribution of avalanche durations $T$ and additional predictions from MFT are discussed in the Supplementary Information, including the following exponent relation: $\alpha=(\kappa-1) \phi+1$ (refs 23,24$)$. Note that the quantities in the first four rows of the table refer to avalanches that are small compared with the size of the system. As before, $v^{*} \equiv 1 /((1+\varepsilon) c)$, see Fig. 1

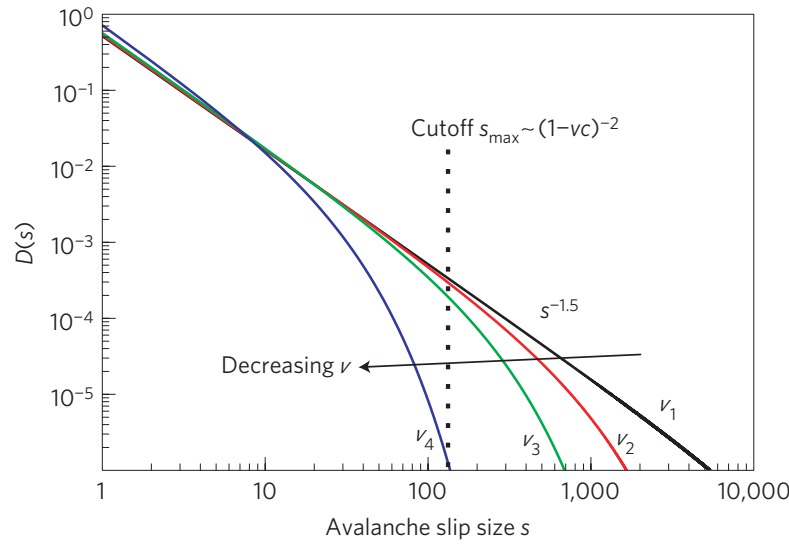

Figure 2 | Analytically predicted mean field slip avalanche size distribution $D(s)$ for decreasing granular number fractions $v$. Here $v_{1} c=0.99$ (black), $v_{2} c=0.95$ (red), $v_{3} c=0.9$ (green) and $v_{4} c=0.7$ (blue), for $\varepsilon=0, V \rightarrow 0$, and $c=1$. Note that the power law $D(s) \sim s^{-3 / 2}$ extends to the largest sizes for the highest number fraction, that is, as $v c \rightarrow 1$. The power law agrees with experiments ${ }^{28}$, see Table 1. The maximum ('cutoff') avalanche size scales as $s_{\max } \sim(1-v c)^{-2}$. The distributions $D(s)$ are normalized. The avalanche slip size $s$ is in arbitrary units. (For $\varepsilon>0, D(s)$ develops a bump ${ }^{22}$.)

sheared plastic solids ${ }^{19-21}$.) (3) Concepts such as anisotropy in the contact distribution and/or the force-intensity distribution, as well as anisotropy and rotation of particles are neglected. (4) Rather than calculating the exact sequence of slips we compute statistical properties on long length scales, such as the probability distributions for large avalanche sizes, long avalanche durations and related measures, as a function of the key model parameters $v$ and $\varepsilon$. (The dependence on the shear rate $V$ is discussed in the Supplementary Information.)

The lattice occupation variable, $o_{i}=1$ if site $i$ is occupied and $o_{i}=0$ if site $i$ is vacant. $u_{i}(t)$ is the total amount of slip (the 'displacement discontinuity') at site $i$ and time $t$. In MFT, $u_{i}(t)$ couples to the average cumulative slip, $\sum_{m=1}^{N}\left(o_{m} u_{m}(t)\right) / N$. The total shear stress $\tau_{i}(t)$ at site $i$ and at time $t$ is

$$
\tau_{i}(t)=J o_{i}\left[\left(\sum_{m=1}^{N} o_{m} u_{m}(t) / N\right)-u_{i}(t)\right]+K_{L}\left(V t-u_{i}(t)\right)
$$

The bulk acts like a soft spring (with constant $K_{L} \sim 1 / L$ ), coupling the lattice to the boundary ${ }^{15}$. A failing site $i$ slips by an

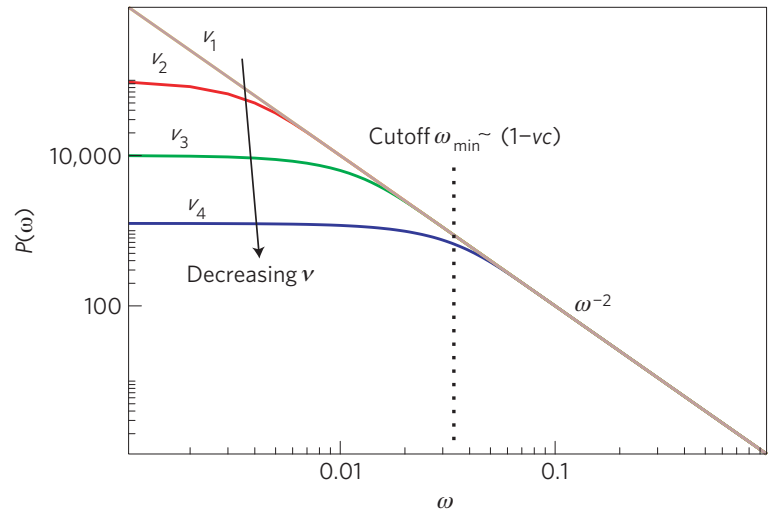

Figure 3 | Illustration of the mean field power spectra at different granular number fractions $\boldsymbol{v}$. Here $v_{1}>v_{2}>v_{3}>v_{4}$ with $\varepsilon \rightarrow 0$ and $V \rightarrow 0$. Note that at high number fraction the power spectrum scales as $P(\omega) \sim \omega^{-2}$ (as seen in experiments ${ }^{10-12,28}$ ) for frequencies $\omega$ above a low-frequency cutoff $\omega_{\min }$. The low-frequency cutoff to the power law region scales as $\omega_{\min }(v, \varepsilon=0) \sim(1-v c)$, with $c \approx 1$. For decreasing number fractions an increasing range of low frequencies $\omega<\omega_{\min }$ scales as $\omega^{0}=$ const (white noise), in agreement with simulations ${ }^{14,17,18}$.

amount $\Delta u_{i}=\Delta \tau_{i} o_{i} /\left(J+K_{L}\right)$. The associated local stress drop is $\Delta \tau_{i} \equiv\left(\tau_{s, i}-\tau_{a, i}\right)$, or, for a weakened cell, $\Delta \tau_{i} \equiv\left(\tau_{d, i}-\tau_{a, i}\right)$. The coupling $J$ redistributes $\Delta \tau_{i}$ to the other sites $k \neq i$, thereby increasing their stress by $\Delta \tau_{k}=o_{i} o_{k}\left|\Delta \tau_{i}\right| J /\left[\left(J+K_{L}\right) N\right]$. We call $c \equiv J /\left(J+K_{L}\right) \approx 1-O(1 / L) \approx 1$ (refs 15,22). The total redistributed stress is then (see Supplementary Information)

$$
\sum_{k=1, k \neq i}^{N} \Delta \tau_{k}=v c\left|\Delta \tau_{i}\right| \leq\left|\Delta \tau_{i}\right|
$$

It is smaller than the original stress drop $\left|\Delta \tau_{i}\right|$ by a factor $v c=\left(N_{\text {occ }} / N\right) J /\left(J+K_{L}\right) \leq 1$. Equation (2) means that voids in granular materials effectively 'dissipate' a fraction $1-v c$ of the released stress. Consequently, on average, the avalanches are smaller when the grains are packed less densely.

For high number fractions $(v c \approx 1, \varepsilon \approx 0, V \rightarrow 0)$ MFT predicts that the distribution $D(s)$ of slip avalanche sizes $s \equiv \sum_{m=1}^{N}\left(\Delta u_{m}\right)$ decays for large $s$ as $D(s) \sim s^{-\kappa} F\left(s / s_{\max }\right)$ (ref. 22). The MFT exponent $\kappa=3 / 2$, and the cutoff function $F$ are expected to be the same for many different materials ('universal'). $F\left(s / s_{\max }\right) \approx$ constant for $s \ll s_{\max }$ and $F\left(s / s_{\max }\right)$ decays exponentially for $s \gg s_{\max }$. The cutoff 


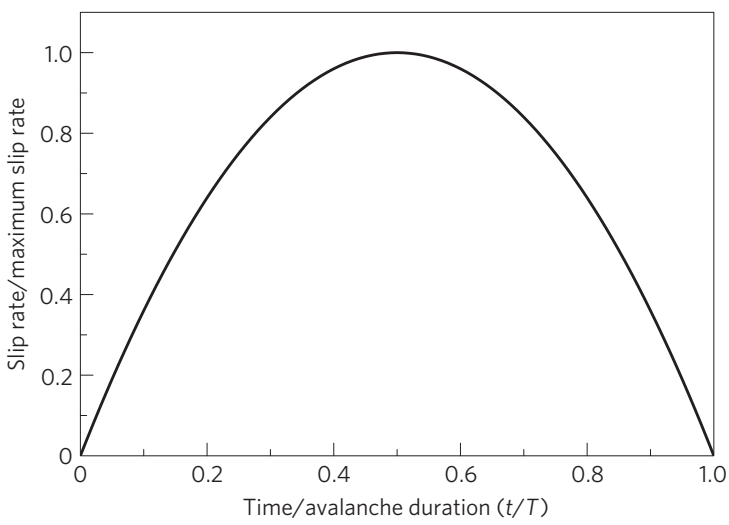

Figure 4 | Rescaled average temporal slip rate profile, or temporal mean field avalanche 'shape'. The profile is obtained from the average total slip rate $\langle V(t, T)\rangle$ during an avalanche versus time $t$ (the 'source time function'), averaged over all avalanches with the same duration $T$. This function is a prediction for avalanches in the power law scaling regime $s<s_{\max }(v, \varepsilon, V)$ of $D(s)$, for $v c$ close to 1 and $\varepsilon$ close to 0 and $V \rightarrow 0$. In MFT $\langle V(t, T)\rangle \sim A x(1-x)$ (refs 23-26), where $x \equiv t / T$. $A$ is a nonuniversal constant ( $A=4$ in the figure), see ref. 26 and the Supplementary Information. A parabola was recently seen in experiments ${ }^{28}$.

$s_{\max }$ scales with the number fraction $v$ as $s_{\max }(v c, \varepsilon=0, V \rightarrow 0) \sim$ $(1-v c)^{-2}$ (see Fig. 2).

Similarly, the power spectrum (the absolute square of the Fourier transform) of the total slip rates $V(t)=\sum_{m=1}^{N} \mathrm{~d} u_{m}(t) / \mathrm{d} t$ scales in MFT as $P_{\omega}(\omega) \sim \omega^{-2} P_{1}\left(\omega / \omega_{\min }\right)$ (refs 21,22$)$. Here $P_{1}\left(\omega / \omega_{\min }\right) \approx$ constant for frequency $\omega>\omega_{\min }(\nu, \varepsilon, V)$ and lowfrequency cutoff $\omega_{\min }(v, \varepsilon=0, V \rightarrow 0) \sim(1-v c)$ (see Fig. 3). Further predictions, such as the $V$ - and $\varepsilon$-dependence of $D(s)$ and $P_{\omega}(\omega)$, and possible experimental tests are discussed in the Supplementary Information.

Much can be learned from the average temporal slip rate profile, $\langle V(t, T)\rangle$ (see Fig. 4). It is the total slip rate versus time $t$ during an avalanche, averaged over all avalanches with same duration $T$. In MFT it is a parabola $\langle V(t, T)\rangle \sim t(T-t) / T$ (refs 23-25). Aharonov and Sparks fitted a sine function to their simulation results, but a parabola may fit equally well ${ }^{14,17,18}$. Many other scaling predictions can be computed from MFT (refs 15,23,24, 26,27). Table 1 shows good agreement of the MFT results with experiments and simulations.

In the solid-like regime $\left(v>v^{*}(\varepsilon)\right.$ with $\left.v^{*}(\varepsilon) \equiv 1 /(c(1+\varepsilon))\right)$, MFT predicts mode switching ${ }^{22}$ : the material flip-flops between time periods with power law distributed avalanches, and periods with 'quasi-periodically' recurring macroscopically large events with only small precursors ('stick-slip'). This behaviour is also seen in experiments ${ }^{28}$. The mean durations of these periods depend on $v, \varepsilon$ and details of the system ${ }^{22}$. As $\varepsilon \rightarrow 0$ and $v c \rightarrow 1$ all the time is spent in the power law phase.

In the fluid-like regime $\left(v<v^{*}(\varepsilon)\right)$, MFT predicts only small avalanches without mode-switching ${ }^{22}$. This regime resembles the 'fluid' phase in experiments and simulations at low number fractions ${ }^{14,17,18}$. In the simulations, the power spectrum $P(\omega) \sim \omega^{0}$ ('white noise') in this phase. The analogous quantity in our model is the power spectrum of the time series of $\Sigma_{m} K_{L}\left(V t-u_{m}(t)\right)$, which scales as $P(\omega) \sim \omega^{0}$ for $v \ll v^{*}(\varepsilon)$, in agreement with simulations and experiments. In all parameter regimes the slip locations are randomly spread throughout the shear band, as seen in experiments ${ }^{12}$ and simulations ${ }^{14,17,18}$.

To conclude, an analytical MFT model is developed which yields a new phase diagram (Fig. 1) for the avalanche statistics of sheared granular materials. The tuning parameters are the grain number fraction $v$, the frictional weakening $\varepsilon$, and the shear rate $V$. Table 1 demonstrates agreement between results from MFT, experiments, and numerical simulations.

Mapping to other systems: remarkably, the universal aspects of avalanches in sheared granular materials at high $v$ (this paper), dislocation slips in solids ${ }^{19-21}$, and earthquakes ${ }^{15,16,22,26,27,29}$ can all be modelled by variants of the same MFT (refs 15,16,22,27), and are thus expected to have the same critical exponents. (The variants describe the undiluted case, that is, $v=1$.) A similar connection between avalanches in granular materials and magnetic domain walls has been pointed out ${ }^{10,11}$. Connections between granular avalanches and earthquakes have been discussed ${ }^{6,16}$. Observations and experiments on these systems yield critical exponents with overlapping error bars. Further studies of the spatial correlations in finite dimensions will aid in the challenging quest for a simple unifying theory underlying the dynamics of these different systems.

\section{Received 22 July 2009; accepted 22 February 2011; published online 27 March 2011}

\section{References}

1. Depken, M., von Saarlos, W. \& van Hecke, M. Continuum approach to wide shear zones in quasi-static granular matter. Phys. Rev. E 73, 031302 (2006).

2. MiDi, G. D. R. On dense granular flows. Eur. Phys. J. E 14, 341-365 (2004).

3. Jaeger, H., Nagel, S. R. \& Behringer, R. P. Granular solids, liquids, and gases. Revs. Mod. Phys. 66, 1259-1273 (1996).

4. Liu, A. J. \& Nagel, S. R. Nonlinear dynamics: Jamming is not just cool any more. Nature 396, 21-22 (1998).

5. Cates, M. E., Wittmer, J. P., Bouchaud, J-P. \& Claudin, P. Jamming, Force Chains, and Fragile Matter. Phys. Rev. Lett. 81, 1841-1844 (1998).

6. Daniels, K. E. \& Hayman, N. W. Force chains in seismogenic faults visualized with photoelastic granular shear experiments. J. Geophys. Res. 113, B11411 (2008).

7. Zhang, J., Majmudar, T. S., Tordesillas, A. \& Behringer, R. P. Statistical properties of a 2D granular material subjected to cyclic shear. Granul. Matter 12, 159-172 (2010).

8. O’Hern, C. S., Langer, S. A., Liu, A. J. \& Nagel, S. R. Force distributions near the jamming and glass transition. Phys. Rev. Lett. 86, 111-114 (2001).

9. Majmudar, T. S. \& Behringer, R. P. Contact force measurements and stress induced anisotropy in granular materials. Nature 435, 1079-1082 (2005).

10. Petri, A. et al. Stochastic dynamics of a sheared granular medium. Eur. Phys. J. B 64, 531-535 (2008).

11. Baldassari, A. et al. Brownian forces in sheared granular matter. Phys. Rev. Lett. 96, 118002 (2006).

12. Hartley, R. PhD Thesis, Physics, Duke University (2005).

13. Cheng, X. et al. Onset of three-dimensional shear in granular flow. Phys. Rev. Lett. 96, 038001 (2006).

14. Aharonov, E. \& Sparks, D. Shear profiles and localization in simulations of granular materials. Phys. Rev. E 65, 051302 (2002).

15. Fisher, D. S., Dahmen, K. A., Ramanathan, S. \& Ben-Zion, Y. Statistics of earthquakes in simple models of heterogeneous faults. Phys. Rev. Lett. 78, 4885-4888 (1997).

16. Ben-Zion, Y. Collective behavior of earthquakes and faults: Continuum-discrete transitions, progressive evolutionary changes and different dynamic regimes. Rev. Geophys. 46, RG4006 (2008).

17. Aharonov, E. \& Sparks, D. Rigidity phase transition in granular packings. Phys. Rev. E 60, 6890-6896 (1999).

18. Aharonov, E. \& Sparks, D. Stick-slip motion in simulated granular layers. J. Geophys. Res. 109, B09306 (2004).

19. Zaiser, M. Scale invariance in plastic flow of crystalline solids. Adv. Phys. 55, 185-245 (2006)

20. Miguel, M-C. \& Zapperi, S. Fluctuations in plasticity at the microscale. Science 312, 1151-1152 (2006).

21. Dahmen, K. A., Ben-Zion, Y. \& Uhl, J. T. A micromechanical model for deformation in disordered solids with universal predictions for stress-strain curves and related avalanches. Phys. Rev. Lett. 102, 175501 (2009).

22. Dahmen, K. A., Ertas, D. \& Ben-Zion, Y. Gutenberg-Richter and characteristic earthquake behavior in simple mean-field models of heterogeneous faults. Phys. Rev. E 58, 1494-1501 (1998).

23. Sethna, J. P., Dahmen, K. A. \& Myers, C. R. Crackling Noise. Nature 410, 242-250 (2001).

24. Kuntz, M. \& Sethna, J. P. Noise in disordered systems: The power spectrum and Dynamic exponents in avalanche models. Phys. Rev. B 62, 11699-11708 (2000).

25. Papanikolaou, S. et al. Beyond scaling: The average avalanche shape. Nature Phys. doi:10.1038/nphys1884 (2011).

26. Mehta, A. P., Dahmen, K. A. \& Ben-Zion, Y. Universal mean moment rate profiles of earthquake ruptures. Phys. Rev. E 73, 056104 (2006). 
27. Dahmen, K. \& Ben-Zion, Y. in Encyclopedia of Complexity and System Science (eds Marchetti, C. \& Meyers, R.) (Springer, 2009).

28. Hayman, N. W., Ducloue, L., Foco, K.L. \& Daniels, K.E. Granular controls on periodicity of stick-slip events: kinematics and force-chains in an experimental fault. Pure Appl. Geophys. doi:10.1007/s00024-011-0269-3 (2011).

29. Chen, K., Bak, P. \& Obukhov, S. P. Self-organized criticality in a crack-propagation model of earthquakes. Phys. Rev. A 43, 625-630 (1991).

\section{Acknowledgements}

We thank R. Behringer, B. Chakroborty, K. Daniels, T. Earnest, J. P. Sethna and

G. Tsekenis for useful discussions, and NSF DMR 03-25939 (MCC), MGA, the USC
Earth Sciences Department, the UCSB Kavli Institute of Theoretical Physics, and the Aspen Center of Physics for support and hospitality.

\section{Author contributions}

All authors contributed extensively to the work presented in this paper.

\section{Additional information}

The authors declare no competing financial interests. Supplementary information accompanies this paper on www.nature.com/naturephysics. Reprints and permissions information is available online at http://npg.nature.com/reprintsandpermissions. Correspondence and requests for materials should be addressed to K.A.D. 